\title{
Control of Green Peach Aphid (Myzus Persicae) by Combination of Plant Oil Formulations and Low-dosed Imidacloprid
}

\author{
You Ri Yang ${ }^{1}$, Seon Hwa Kim ${ }^{1}$, Myung Ryeol Park${ }^{1}$, Iksoo Kim² and In Seon Kim ${ }^{1 *}$ \\ ${ }^{1}$ Division of Applied Bioscience and Biotechnology, ${ }^{2}$ Division of Plant Biotechnology, Institute of Agricultural Science and \\ Technology, College of Agriculture and Life Sciences, Chonnam National University, Gwangju 500-757, South Korea \\ (Received August 24, 2010, Accepted September 17, 2010)
}

\begin{abstract}
Aphids are one of the major pests in agricultural crops. A number of synthetic pesticides have been used for control of aphids in agriculture, but increasing public concems over their adverse effects on the environment have required more environmentally-friendly methods for pest management. In this study, we examined plant oil formulations for the control of green peach aphid (Myzus persicae). Oil formulations were prepared by hydrolyzing the plant oils in ethanolic KOH solution and diluted at the rate of 1:500 for aphid control. The oil formulations showed aphid mortalities ranging from 24.44 to $43.33 \%$ in vitro. Significantly increased aphid mortalities were observed by the treatment of oil formulations combined with low-dosed imidacloprid. No significant difference in the aphid mortality was observed between the oil formulations. Mass spectrometry analyses of aphids treated with the low dosedimidacloprid plus the plant oil formulations detected similar concentrations of imidacloprid between the treatments. In field trial bioassays against aphids, significantly decreased aphid population were observed in the pepper plants treated with soybean oil formulation combined with the low-dosed imidacloprid, while aphid population dramatically increased in the pepper plants treated with the low-dosed imidacloprid alone. These results suggested that the plant oil formulations can be used as an environmentally-friendly method for enhancing the insecticidal effectiveness, which may play a role in reducing the use of synthetic pesticide in agriculture.
\end{abstract}

Key Words: Aphid, Imidacloprid, Insecticide, Organic farming, Plant oil

\section{INTRODUCTION}

Aphids are one of the major insect pests in agriculture. About 100 aphid species are known to cause damages to over 4,000 species of crop plants throughout the world (Dixon, 1987). Aphids feed on plant nutrients, resulting in curled and deformed leaves, and cause a variety of plant pathogens that grow on sugars secreted by aphids (Moran and Thomson, 2001). Aphids are also the causes of many viral diseases, including the cucumber mosaic virus and pot virus, in several crops (Sylvester, 1989; James and Perry, 2004). Although a number of synthetic insecticides have been widely

\section{*연락저자:}

Tel: +82-62-530-2131 Fax: +82-62-530-2139

E-mail: mindzero@chonnam.ac.kr used to control aphids, their intensive use is of the concerns due to the potential side effects on the environment. Additionally it is a worry for pest management scientists to control pesticide-resistant insects (Foster et al., 2000, 2003). Thus, researches have focused on the development of alternative control methods in an effort to reduce the use of synthetic insecticides in agriculture.

Organic farming toward the use of more environmentally safe approach in agriculture has encouraged many scientists to develop new pesticide formulations in order to reduce the use of synthetic pesticides (Kim and Kim, 2009). New trends in crop protection have been triggering the search for not only harmless pesticides or pesticides with very low toxicity and for also ways to reducing the use of 
agrochemicals (Martín-López et al., 2006). One of the ways to reduce the use of synthetic chemicals is to use low-dosed pesticides combined with synergistic agents for crop protection. Application of commercial plant-derived oils is an example approach as a synergistic agent of chemical insecticides (Papachristos et al., 2004). Commercial plant oils have been accepted as a recommended product for pest management program in agricultural field (Ayvaz et al., 2010). One of main advantages in using plant oils for pest control is that they discourage insects from developing resistance to them (Kaloshians and Walling, 2005). In addition, they are non-toxic to vertebrates and degradable easily in the environment. Thus, the use of plant oils is expected to be an environmentally-friendly approach to pest management program in Korea. However, little study on the evaluation of plant oils-derived formulation for control of aphids has been conducted in vitro and in vivo, although limited botanical insecticide is available in organic farming in Korea. In the present study we carried out the aphicidal activities of commercial plant oil formulations combined with a low-dosed imidacloprid.

\section{MATERIALS AND METHODS}

\section{Chemicals}

Imidacloprid analytical standard was purchased from Dr. Ehrenstorfer (Ausburg, Germany). Conido (SC 8\%), an imidacloprid formulation, was kindly provided by Bayer CropScience Korea. The solvents used in this study were of HPLC grade and were purchased from Fisher Scientific (Pittsburgh, PA). Plant oils (soybean, canola, grape-seed and olive) were commercially available elsewhere in Korea. All chemicals used were of analytical grade, unless otherwise stated.

\section{Plant oil formulation}

Plant oil formulations were prepared by hydrolyzing them under basic conditions. For this, each of the plant oils $(92.6 \mathrm{~g} / \mathrm{L})$ was hydrolyzed with three equivalent of $\mathrm{KOH}$ in ethanol at room temperature. After completely and vigorously mixed for $2 \mathrm{~h}$, the oil solutions were adjusted to approximately $\mathrm{pH} 10.8$ by addition of $5.8 \mathrm{M} \mathrm{HCl}$ in ethanol, and the resulting solutions were used as the plant oil formulation.

\section{Laboratory bioassay for aphid mortality}

Laboratory bioassays of the plant oil formulations against green preach aphid (M. persicae) were performed in 2-week-old Chinese cabbage as described previously (Kim et al., 2008). Chinese cabbage seeds were disinfected with $70 \%$ ethanol for $1 \mathrm{~min}$ and then rinsed several times with sterile distilled water. The seeds were then planted in commercial compost in square plastic insect-breeding dishes $(80 \mathrm{~mm} \times 80 \mathrm{~mm} \times 100$ $\mathrm{mm}$ ) and grown in a growth chamber at $25 \pm 2^{\circ} \mathrm{C}$ and $60 \pm 5 \%$ relative humidity with a $16 \mathrm{~h}$ light: $8 \mathrm{~h}$ dark photoperiod for 2 weeks. Ten M. persicae were reared on the Chinese cabbage leaves and allowed to produce progeny for $48 \mathrm{~h}$ in a greenhouse. The adult aphids were then removed from the leaves, and each dish contained 18 to 24 2nd-instar nymphs. The oil formulations were diluted with water at the rate of 1:500 (hereafter 'OF500') and sprayed onto the nymphs on the Chinese cabbage leaves using a small handheld sprayer. For the bioassay of imidacloprid, Conido $^{R}$ was sprayed onto the nymphs at a recommended field application rate $(40 \mathrm{mg} / \mathrm{L})$ for vegetables. For the bioassays of low-dosed imidacloprid, Conido ${ }^{R}$ was diluted in OF500 from the recommended dose to reach the application doses of 4.0, 1.6 and $0.8 \mathrm{mg} / \mathrm{L}$ and sprayed onto the nymphs as described above. Aphid mortality was calculated using Abbott's formula $24 \mathrm{~h}$ after application as previously (Abbott, 1925), where mortality $(\%)=[$ (number of live insects on control leaves - number of live insects on treated leaves)] $\times 100 /$ (number of live insects on control leaves). All experiments were repeated three times, unless otherwise stated. Statistical analyses was subjected to Duncan's multiple range test $(\mathrm{p}<0.05)$ using SAS program.

\section{Field trial bioassay for aphid mortality}

Field trial bioassays of the oil formulations were performed against naturally occurring $M$. persicae in a pepper greenhouse in Nampyung, Chonnam Province, Republic of Korea, in July 2009. The greenhouse has cultivated Korean hot pepper plants for at least 7 years. The greenhouse suffered serious $M$. persicae damage during the season. Ten plants with high aphid populations were randomly selected in the greenhouse and subjected to aphid mortality assays. The plants were $2.0 \mathrm{~m} \times 2.0 \mathrm{~m}$ apart from each other not 
to have aerosol migration at the sides of each pepper plant while spraying. Soybean oil formulation was selected as a typical application for aphid mortality assay since it is typical common plant oil commercially available in Korea. Imidacloprid $\left(\right.$ Conido $^{R}$ ) was diluted in soybean oil OF500 solution from the recommended dose to reach the application dose of 0.8 $\mathrm{mg} / \mathrm{L}$, as described above, and sprayed onto pepper plants using a farmer's handheld sprayer. The number of aphids was counted before and $24 \mathrm{~h}$ after application by sampling from three plants for control samples and ten plants for treated samples. Aphid mortality was investigated as follows: mortality $(\%)=[1-$ (number of live aphids after application/ number of live aphids before application)] × 100 .

\section{Detection of imidacloprid in aphids}

Imidacloprid was determined by using quadruple time-of-flight mass spectrometry. Thirty dead-aphids treated with low-dosed imidacloprid combined with OF500 were gently washed with $70 \%(\mathrm{v} / \mathrm{v})$ ethanol solution to remove imidacloprid on the aphid surface and dried in the air. The aphids were then frozen in liquid nitrogen and macerated using a table homogenizer. The homogenates were suspended in $5 \mathrm{~mL}$ of $50 \%(\mathrm{v} / \mathrm{v})$ aqueous methanol and extracted by an ultra-sonicator for $30 \mathrm{~min}$. The extracts were centrifuged at $12,000 \times \mathrm{g}$ and the resulting supernatants were filtered through a $0.2 \mu \mathrm{m}$ membrane filter. The filtrates were used for the detection of imidacloprid in aphids.

\section{Instrumentals}

Gas chromatography mass spectrometry (GC/MS) was used for fatty acid analysis of OF500. GC/MS was a Shimadzu model QP2010 equipped with a DB-5 capillary column (0.25 i.d. $\times 30 \mathrm{~m}, 1.0 \mu \mathrm{m}$ film thickness). The carrier gas was helium and the flow rate was $1.0 \mathrm{~mL} / \mathrm{min}$. Injection was done in a split mode of 10:1. The column temperature was set for 4 min at $120^{\circ} \mathrm{C}$, followed by a ramp rate of $5^{\circ} \mathrm{C} / \mathrm{min}$ to $300^{\circ} \mathrm{C}$, holding for $10 \mathrm{~min}$. The injector temperature was $280^{\circ} \mathrm{C}$. GC/MS was performed in electron impact mode at $70 \mathrm{ev}$. For the detection of imidacloprid in aphids, quadruple time-of-flight mass spectrometry (Q-TOF MS) was used at extracted ion chromatogram (XIC) mode, using the following operation parameters: capillary voltage: $4100 \mathrm{~V}$; nebulizer pressure: 11.6 psig; collision cell RF: $200 \mathrm{Vpp}$; dry heater: $200^{\circ} \mathrm{C}$; dry gas flow rate: $8.0 \mathrm{~L} / \mathrm{min}$. Q-TOF MS was a Bruker Daltonics TOF mass spectrometer connected with a Dionex model P680 HPLC. The HPLC column was a Nova-Pak C18 stainless steel column $\left(2.0{ }^{\circ} \mathrm{C} 150 \mathrm{~mm}\right)$. The mobile phase consisted of $50 \%$ aqueous acetonitrile acidified with trifluoroacetic acid, and the flow rate was $0.5 \mathrm{~mL} / \mathrm{min}$. The HPLC system was interfaced to Q-TOF MS equipped with electrospray ionization interface in positive ion mode. Accurate mass spectra were scanned in a range of 50 to 800 .

\section{RESULTS}

GC/MS analysis of OF500 detected palmitic (C16:0), stearic acid (C18:0), oleic acid (C18:1) and linoleic acid (C18:2) as the main fatty acids of plant oils (Fig. 1). Oleic acid and linoleic acid were found to be the highest composition among them, accounting for more than $80 \%$ of total fatty acid. The ratio of saturated fatty acid to unsaturated fatty acid ranged from 0.10 to 0.16 , which was not significantly different between the plant oils. The aphicidal activity of OF500 against $M$. persicae were $36.67 \%$ for soybean oil, $43.33 \%$ for grape-seed oil, $34.45 \%$ for canola oil and $24.44 \%$ for olive oil (Table 1), exhibiting the highest mortality for grape-seed oil. Increased aphid populations were observed in the samples treated with water as a negative control.

Imidacloprid exhibited $100 \%$ mortality of M. persicae at the recommended field application rate, but significantly decreased mortality was observed by the low doses of imidacloprid, exhibiting about 60 and $47 \%$ mortality at $1 / 10$ and $1 / 40$ doses of the recommended rate, respectively (Table 2). About 14\% mortality of M. persicae was observed in imidacloprid treatment at $1 / 80$ dose of the recommended rate. The dose-dependent mortality of $M$. persicae suggested that the mortality was due to the toxicity of imidacloprid.

The aphid mortality of the low-dosed imidacloprid was largely increased by adding OF500 treatment. The mortality of $M$. persicae by the low dosed-imidacloprid combined with soybean oil OF500 ranged from about 81 to $100 \%$ (Table 3), which was significantly higher than the mortality of 13.7 to $60.0 \%$ by the lowdosed imidacloprid alone. The aphid mortality of the 


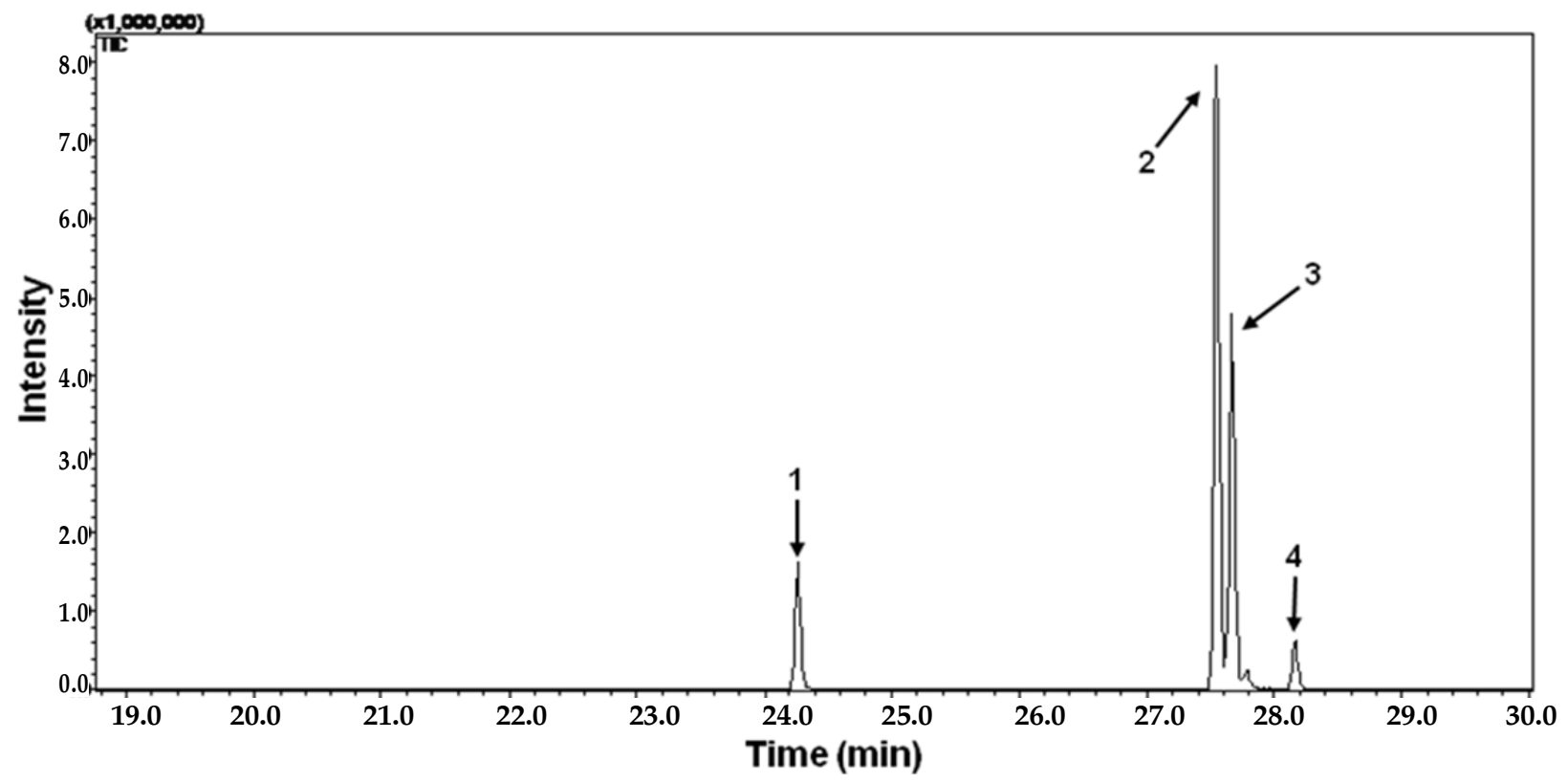

Fig. 1. Typical GC/ MS spectrum of fatty acids of the soybean oil OF500. Peaks: 1. Palmitic acid methyl ester, 2. Linoleic acid methyl ester, 3. Oleic acid methyl ester, 4. Stearic acid methyl ester.

Table 1. Aphid mortalities of plant oil formulations

\begin{tabular}{cc}
\hline- & Aphid mortality $(\%)^{*}$ \\
\hline Control & $0.00 \pm 0.00$ \\
Soybean & $36.67 \pm 11.55$ \\
Grape-seed & $43.33 \pm 15.28$ \\
Canola & $34.45 \pm 25.01$ \\
Olive & $24.44 \pm 12.01$ \\
\hline
\end{tabular}

* The data are means \pm SD of triplicate.

Table 2. Aphid mortalities of imidacloprid at different concentrations

\begin{tabular}{cc}
\hline Treated dose $(\mathrm{mg} / \mathrm{kg})$ & Aphid mortality $(\%)^{*}$ \\
\hline Control & $0.00 \pm 0.00$ \\
$40.0^{* *}$ & $100.00 \pm 0.00$ \\
4.0 & $60.00 \pm 17.32$ \\
1.6 & $47.02 \pm 17.16$ \\
0.8 & $13.70 \pm 5.48$ \\
\hline
\end{tabular}

* The data are means \pm SD of triplicate.

** Recommended field application rate

Table 3. Aphid mortalities of imidacloprid combined with soybean oil OF500

\begin{tabular}{cc}
\hline Imidacloprid $(\mathrm{mg} / \mathrm{kg})+$ soybean oil & Aphid mortality $(\%)^{*}$ \\
\hline Control & $0.00 \pm 0.00$ \\
$40.0^{* *}$ & $100.00 \pm 0.00$ \\
4.0 & $100.00 \pm 0.00$ \\
1.6 & $100.00 \pm 0.00$ \\
0.8 & $81.07 \pm 9.63$ \\
\hline
\end{tabular}

\footnotetext{
* The data are means \pm SD of triplicate.

** Recommended field application rate.
} 
Table 4. Aphid mortalities of imidacloprid combined with canola oil OF500

\begin{tabular}{cc}
\hline Imidacloprid $(\mathrm{mg} / \mathrm{kg})+$ canola oil & Aphid mortality $(\%)^{*}$ \\
\hline Control & $0.00 \pm 0.00$ \\
$40.0^{* *}$ & $100.00 \pm 0.00$ \\
4.0 & $93.33 \pm 11.55$ \\
1.6 & $86.30 \pm 15.17$ \\
0.8 & $81.67 \pm 10.10$ \\
\hline
\end{tabular}

* The data are means \pm SD of triplicate.

** Recommended field application rate.

Table 5. Aphid mortalities of imidacloprid combined with grape-seed oil OF500

\begin{tabular}{cc}
\hline Imidacloprid $(\mathrm{mg} / \mathrm{kg})+$ grape-seed oil & Aphid mortality $(\%)^{*}$ \\
\hline Control & $0.00 \pm 0.00$ \\
$40.0^{* *}$ & $100.00 \pm 0.00$ \\
4.0 & $100.00 \pm 0.00$ \\
1.6 & $96.67 \pm 5.77$ \\
0.8 & $66.67 \pm 4.41$ \\
\hline
\end{tabular}

* The data are means \pm SD of triplicate.

** Recommended field application rate.

Table 6. Aphid mortalities of imidacloprid combined with olive oil OF500

\begin{tabular}{cc}
\hline Imidacloprid $(\mathrm{mg} / \mathrm{kg})+$ grape-seed oil & Aphid mortality $(\%)^{*}$ \\
\hline Control & $0.00 \pm 0.00$ \\
$40.0^{* *}$ & $100.00 \pm 0.00$ \\
4.0 & $100.00 \pm 0.00$ \\
1.6 & $96.67 \pm 5.77$ \\
0.8 & $66.67 \pm 4.41$ \\
\hline
\end{tabular}

\footnotetext{
* The data are means \pm SD of triplicate.

** Recommended field application rate.
}

low dosed-imidacloprid combined with canola oil OF500 ranged from about 81.7 to $93.3 \%$ (Table 4), comparable to the mortality by the low-dosed imidacloprid alone. The low dosed-imidacloprid combined with grape-seed oil OF500 or olive oil OF500 exhibited aphid mortalities ranging from about 67 to $100 \%$ (Table 5 and 6).

In greenhouse experiment, aphids were sprayed with imidacloprid at $1 / 40$ dose of the recommended rate combined with soybean oil OF500, which gave about 50.6 to $87.7 \%$ mortalities (Fig. 2). Lower mortalities of aphids in the field trial than those in the laboratory evaluations may be due to aphid reside under plant leaves at full maturity. The control

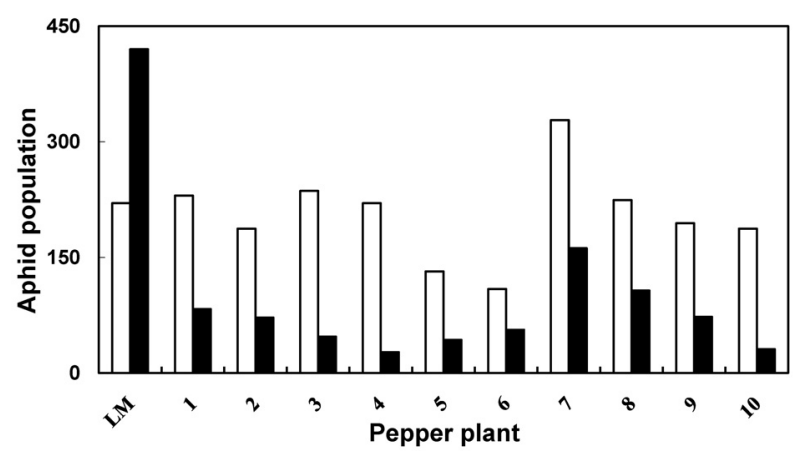

Fig. 2. Changes in aphid population before ( $\square$ ) after ( $\square$ ) spraying a low dose of imidacloprid combined with or without the soybean oil OF500 in field bioassays against green peach aphids. LM represents the low dose of imidacloprid alone. 

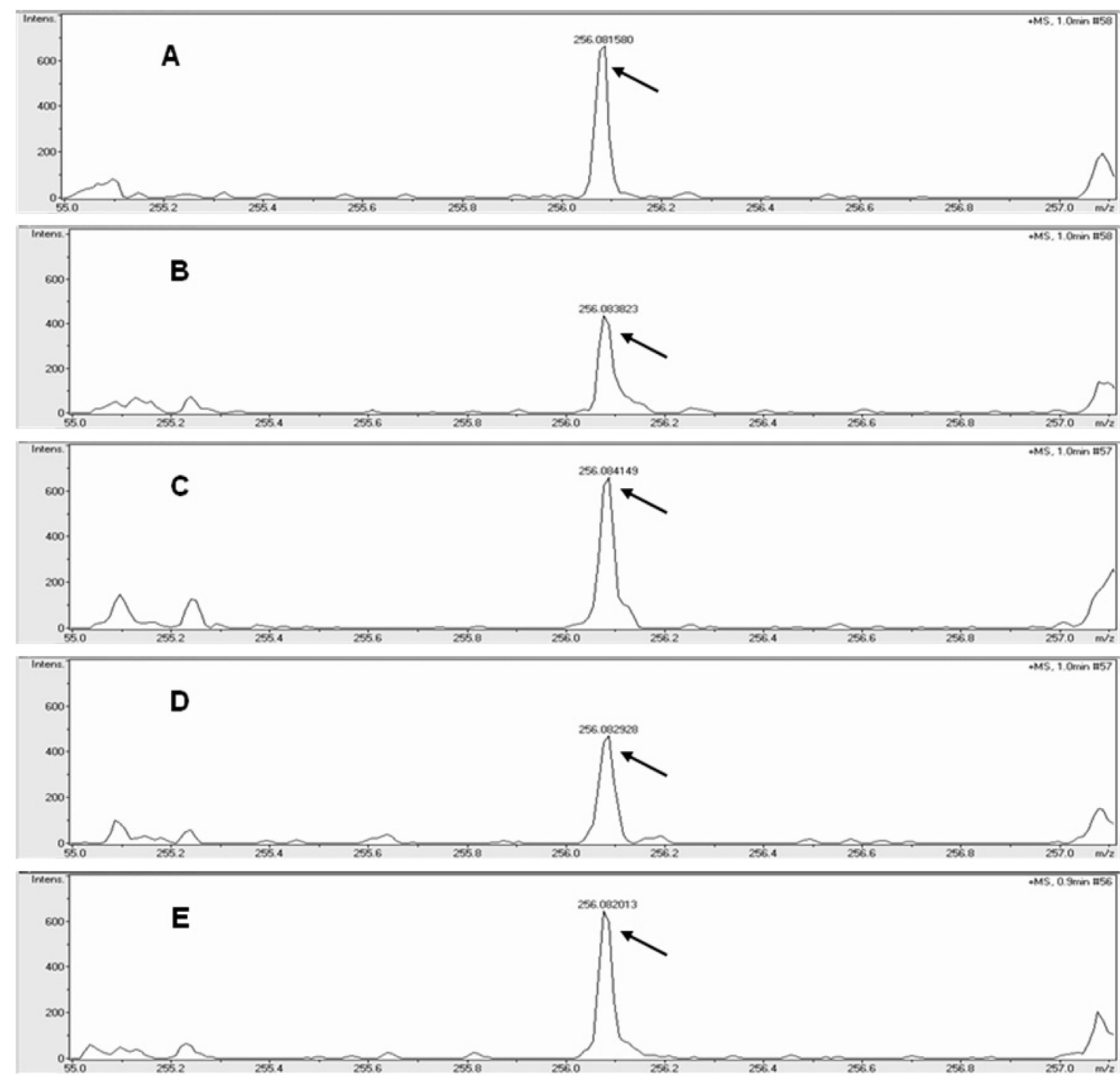

Fig. 3. Typical TOF-MS spectra of imidacloprid extracted from aphids treated with a low dose of imidacloprid alone (A) and the low-dosed imidacloprid combined with soybean oil OF500 (B), canola oil OF500 (C), grape-seed oil PF500 (D) and olive oil OF500 (E). The arrow symbols represent the peaks of imidacloprid detected by TOF-MS.

efficiencies of the combined mixture lasted 5 to 7 days. No phytotoxic symptoms were observed by application of OF500.

In order to investigate if the increased aphid mortality of low-dosed imidacloprid was due to more penetration and accumulation of imidacloprid in aphid, aphids were extracted with organic solvents and analyzed by mass spectrometry (Fig. 3). TOF-MS analysis at XIC mode detected about $0.6 \mathrm{mg} / \mathrm{L}$ of imidacloprid in aphids treated with the low-dosed imidacloprid combined with soybean oil OF500. About $1.0 \mathrm{mg} / \mathrm{L}$ of imidacloprid was detected in aphids treated with the low-dosed imidacloprid combined with canola oil OF500 or olive oil OF500, which was close to the concentration found in aphids treated with the low-dosed imidacloprid alone. TOF-MS detected about $0.7 \mathrm{mg} / \mathrm{L}$ of imidacloprid in aphids treated with low-dosed imidacloprid combined with grape-seed oil OF500.

\section{DISCUSSION}

The intensive use of synthetic pesticides in agriculture has become an environmental concern due to 
their toxicities and persistence in the environment. Thus, Korea government has driven environmentallyfriendly agriculture (EFA) program since 1995. The EFA program has been established with the goal of decreasing the use of synthetic pesticides by $40 \%$. This large reduction in the use of synthetic pesticides could be of negative impacts on agriculture, since crop yields may be significantly decreased due to plant pathogens and insect pests that have been managed successfully by synthetic pesticides (Oerke and Dehane, 2004). Thus, alternative approaches are required for pest management, particularly in organic farming. In the present study, we examined plant oil-derived formulations in order to develop a method as more environmentally safe approach for the control of green peach aphids.

Mortality of aphid treated with low doses of imidacloprid was significantly enhanced in vitro by the plant oil formulations as compared to the mortality by low-dosed imidacloprid alone. Results of greenhouse experiments indicated that the plant oil formulations may provide high mortality levels of aphids under field conditions. TOF-MS analyses demonstrated that the increased aphid mortality would not be due to the increased penetration or accumulation of imidacloprid, since the concentration of imidacloprid found in aphids treated with the combined mixture was similar to the concentration found in aphids treated with low-dosed imidacloprid alone. The synergistic effects of the plant oil formulations on aphid mortality may be an explanation for the increased mortality, as demonstrated earlier (Puri et al., 1994; Horowitz et al., 1997; Rao and Dhingra, 1997; Liu and Stansely, 2000). The plant oil formulations are expected to play the roles of wetting, spreading and sticking agents on aphids. One of possible actions of plant oil formulations was suggested to be their asphyxial effects on aphid surface, as described previously a synergistic effect of oils on insect control (Dhingra, 1996). Aphids would be covered with a thin film of oily materials such as fatty acids produced from plant oils. Botanical insecticides, such as nicotine from tobacco leaves, rotenone from derris tree roots, pyrethrum from chrysanthemum flowers and azadirachitin from neem tree have accepted as alternatives to conventional synthetic insecticides (Isman, 1997; Edelson, 2002; Casanova et al., 2005,
Kim et al., 2007, 2009). The plant oil formulations can be also used in order to increase the insecticidal efficacy of botanical insecticides. The plant oil formulations are, therefore, suggested to be an alternative approach for pest management in an effort to reduce the use of synthetic pesticide in agriculture. Further works to examine the oil formulations for aphid control under different agricultural conditions are required. Our results showed clearly that the plant oil formulations are applicable for control of aphid under laboratory and greenhouse conditions.

\section{ACKNOWLEDGEMENT}

This work was supported by a grant from the Environmentally-friendly Agricultural Research Program of the Ministry of Agriculture, Forestry, Fishery and Food, Republic of Korea.

\section{REFERENCES}

Abbott, W. S., 1925. A method of computing the effectiveness of an insecticide, J. Eco. Entomol. 18, 265-267.

Ayvaz, A., Sagdic, O., Karaborklu, S., Ozturk, I., 2010. Insecticidal activity of the essential oils from different plants against three stored-product insects, J. Insect Sci. 10, 1-13.

Casanova, H., Araque, P., Ortiz, C., 2005. Nicotine carboxylate insecticide: Effect of the fatty acid chain length, J. Agric. Food. Chem. 53, 9949-9953.

Dhingra, S., 1996. Effect of different vegetable oils on the toxicity of cypermethrin in mixed formulations on the adults of Mylabris pustulata thunb, J. Entomol. Res. 20, 19-22.

Dixon, A.F.G., 1987. The way of life of aphids: host specificity, speciation and distribution, in: Minks, A.K., Harrewijn, P., (Eds), Aphids, Their Biology, Natural Enemies and Control (2A), Elsevier, Amsterdam, pp. 197-207.

Edelson, J.V., Duthie, J., Roberts, W., 2002. Toxicity of biorational insecticides: activity against the green peach aphid, Myzus persicae (Sulzer), Pest Manag. Sci. 58, 255-260.

Foster, S.P., Denholm, I., Devonshire, A.L., 2000. The ups and down of insecticides resistance in peach- 
potato aphids (Myzus persicae) in the UK, Crop Protect. 19, 873-829.

Foster, S.P., Denholm, I., Thompson, R., 2003. Variations in response to neonicotinoid insecticides in peach-potato aphids, Myzus persicae (Hemiptera: Aphididas), Pest Manag. Sci. 59, 166-173.

Horowitz, A.R., Mendelson, Z., Ishaava, I., 1997. Effect of abamectin mixed with mineral oil on the sweet potato whitefly (Homoptera Aleyrodidae), J. Econ. Entomol. 90, 349-353.

Isman, M.B., 1997. Neem and other botanical insecticides: barriers to commercialization, Phytoparastica. 25, 339-344.

James, C.K.N.G., Perry, K.L., 2004. Transmission of plant viruses by aphid vectors, Mol. Plant Pathol. 5, 505-511.

Kaloshians, I., Walling, L.L., 2005. Hemipterans as plant pathogens, Annu. Rev. Phytopathol. 43, 491-521.

Kim, S.K., Kim, S.R., Choi, M.S., Park, C.E., Kim, Y.C., Kim, K.Y., Whang, K.S., Oh, K.T., Kim, I.S., 2007. Soybean oil-degrading bacterial cultures as a potential for control of green peach aphids (Myzus persicae), J. Microbiol. Biotechnol. 17, 1700-1703.

Kim, H.Y., Lee, H.B., Kim, Y.C., Kim, I.S., 2008. Laboratory and field evaluations of entomopathogenic Lecanicillium attenuatum CNU-23 for control of green peach aphid (Myzus persicae), J. Microbiol. Biotechnol. 18, 1915-1918.

Kim, S.R., Kim, I., Kim, I.S., 2009. Evaluation of a pyrethrum emulsion prepared in food-acceptable components in controlling green epach aphid (Myzus Persicae), J. Korean Soc. Appl. Biol. Chem. 52, 176-179.

Kim, I.S., Kim, I., 2009. Status and future prospects of pest control agents in environmentally-friendly agriculture, and importance of their commercialization, Korean J. Environ. Agric. 28, 301-309.

Liu, T.X., Stansly, P.A., 2000. Insecticidal activity of surfactants and oils against silver leaf whitefly (Bemisia argentifolii) nymphs (Homoptera: Aleyrodidae) on collards and tomato, Pest Manag. Sci. 56, 861-866.

Martín-López, B., Varela, I., Marnotes, S., Cabaleiro, C., 2006. Use of oils combined with low doses of insecticide for the control of Myzus persicae and PVY epidemics, Pest Manag. Sci. 62, 372-378.

Moran, P.J., Thomson, G.A., 2001. Molecular responses to aphid feeding in Arabidopsis in relation to plant defense pathways, Plant Physiol. 125, 1074-1085.

Oerke, E.C., Dehne, H.W., 2004. Safeguarding production-losses in major crops and the role of crop protection, Crop Protect. 23, 275-285.

Papachristos, D.P., Karamanoli, K.I., Stamopoulos, D.C., Menkissoglu-Spiroudi, U., 2004. The relationship between the chemical composition of three essential oils and their insecticidal activity against Acanthoscelides obtectus (Say), Pest Manag. Sci. 60,514-520.

Puri, S.N., Bhosle, B.B., Ilyas, M., Butler, G.D., Henneberry, T.J., 1994. Detergents and plant-derived oils for control of the sweet potato whitefly on cotton, Crop Protect. 13, 45-48.

Rao, G.R., Dhingra, S., 1997. Synergistic activity of some vegetable oils in mixed formulations with cypermethrin against different instars of Spodoptera litura (Fabricius), J. Econ. Entomol. Res. 21, 153-160. Sylvester, E.S., 1989. Viruses transmitted by aphids, in: Minks, A.K., Harrewijn, P., (Eds), Aphids, Their Biology, Natural Enemies and Control (2C), Elsevier, Amsterdam, pp. 65-87. 